\title{
Clinical Preparedness for Cytokine Storm Induced By the Highly Pathogenic H5N1 Influenza Virus
}

\section{Toshihisa Ishikawa*}

RIKEN Yokohama Institute, RIKEN Omics Science Center, 1-7-22 Suehiro-cho, Tsurumi-ku Yokohama 230-0045, Japan

\section{Editorial}

Since the first human case emerged in 1997 in Hong Kong, A (H5N1) viruses have been circulating among avian species and have spread throughout Asia, Europe, and Africa, with sporadic transmission to humans. Hitherto, genetic analysis of human-infected A ( $\mathrm{H} 5 \mathrm{~N} 1)$ virus has revealed that $\mathrm{A}(\mathrm{H} 5 \mathrm{~N} 1)$ virus directly transmitted from birds to human. Nevertheless, the possibility of human-to-human transmission of highly pathogenic avian influenza A (H5N1) viruses is becoming a fear for human health and society. Because humans lack immunity to influenza viruses possessing an H5NA, the emergence of a transmittable H5-HA-possessing virus would probably cause a pandemic $[1,2]$.

Until now, the most dramatic example of defining the pathogenicity of influenza A (H5N1) virus is the higher fatality rate of avian influenza epidemic ( $>50 \%)$ occurred in Southeast Asia in 1997, as compared with the pandemic caused by influenza virus $\mathrm{A}(\mathrm{H} 1 \mathrm{~N} 1)$ in 1918 (5-10\%). The mortality of patients infected with the highly pathogenic influenza A (H5N1) virus increases up to about $60 \%$ more than three days after the onset of symptom [3]. Recent studies have shown that the high fatality rate of avian influenza virus infections is a consequence of an overactive inflammatory response and the severity of infection is closely related with virus-induced "cytokine storm" [4].

The cytokine storm is systemic expression of a healthy and vigorous immune system resulting in the release of more than 150 inflammatory mediators (cytokines, oxygen free radicals, and coagulation factors) [4]. Both pro-inflammatory cytokines (such as TNF- $\alpha$, IL-1, and IL6) and anti-inflammatory cytokines (such as IL-10, and IL-1 receptor antagonist) are elevated in the serum, and the fierce and often lethal interplay of these cytokines takes place. The primary contributors to the cytokine storm are TNF- $\alpha$ and IL-6. The cytokine storm is caused by rapidly proliferating and highly activated T-cells or natural killer (NK) cells. These cells are activated by infected macrophages. In the absence of prompt medical intervention to stop the cytokine storm, the lung will suffer permanent damage and result in death. Many of H5N1-infected patients develop Acute Respiratory Distress Syndrome (ARDS), and deaths will usually result from multi-system organ failure, and not from lung failure. Thus, the "cytokine" storm must be treated and suppressed, otherwise lethality can result.

It has been detected that, mutations of some genes (NS1, PB2, HA and NA) in the A (H5N1) virus are responsible for the cytokine storm, by increasing the viral replication rate, expending the tissue tropism, facilitating the systemic invasion and emerging of resistance against the host antiviral response. It has been shown that Glu92 and Ala149 mutations, and carboxyl-terminal ESEV/EPEV motif of NS1 protein have been implicated as determinants of virulence for the A (H5N1) virus [5]. In addition, Lys627 mutation in PB2 protein, polybasic amino acid mutations in the cleavage region of hemagglutinin (HA) polyprotein, and glycosylation and sialylation mutations in HA and NeuraMinidase (NA) proteins were found to enhance the immunemediated pathology of highly virulent strains of the A (H5N1) virus $[1,5]$.
Early diagnosis and early therapeutic intervention are crucially required to improve the prognosis of the infectious disease. A recent study demonstrates that early initiation of antiviral treatment has a strong influence on the survival of patients with A (H5N1) infection. Kudo et al. [6] have most recently reported that renal replacement therapy using Polymyxin B-immobilized polystyrene fibers (PMX) and Continuous Hemodiafiltration (CHDF) could provide a practical tool to effectively treat $\mathrm{H} 5 \mathrm{~N} 1$-infected patients with acute respiratory distress syndrome (ARDS). PMX (Toray industries Inc, Tokyo, Japan) binds endotoxin and inflammatory cytokines in both in vitro and in vivo studies [7]. PMX hemoperfusion was applied to H5N1-infected patients sequentially using 3 columns at a flow rate of $100 \mathrm{ml} / \mathrm{min}$ for 3 days. Levels of IL-6, IL-8, and TNF- markedly decreased within 24 $\mathrm{h}$ after initiation of PMX hemoperfusion. Accordingly, the $\mathrm{PaO}_{2} / \mathrm{FiO}$ ratio increased to 128 at $24 \mathrm{~h}$ and to 203 at 3 days after initiation of PMX hemoperfusion. Thus, renal replacement therapy using PMX and Continuous Hemodiafiltration (CHDF) provided a practical tool to effectively treat H5N1-infected patients with Acute Respiratory Distress Syndrome (ARDS) [6].

\section{References}

1. Watanabe $Y$, Ibrahim MS, Suzuki $Y$, Ikuta $K(2012)$ The changing nature of avian influenza A virus (H5N1). Trends Miclobiol 20: 11-20.

2. Imai M, Watanabe T, Hatta M, Das SC, Ozawa M, et al. (2012) Experimenta adaptation of an influenza H5 HA confers respiratory droplet transmission to a reassortant $\mathrm{H} 5 \mathrm{HA} / \mathrm{H} 1 \mathrm{~N} 1$ virus in ferrets. Nature 486: 420-428.

3. Nagai $Y$ (2012) A watershed in clinical outcomes of human infections with highly pathogenic H5N1 avian influenza viruses: lessons from case-management in Egypt. Rev Med Virol 22: 351-353.

4. Tisoncik JR, Korth MJ, Simmons CP, Farrar J, Martin TR, et al. (2012) Into the eye of the cytokine storm. Microbiol. Mol Biol Rev 76: 16-32.

5. Us D (2008) Cytokine storm in avian influenza. Mikrobiyol Bul 42: 365-80.

6. Kudo $\mathrm{K}$, Nguyen GB, Manabe $\mathrm{T}$, et al. Clinical preparedness for severe pneumonia with highly pathogenic avian influenza $A(H 5 N 1)$ : Experiences with cases in Vietnam. Respiratory Invest. in press, 2012

7. Shoji $H$ (2003) Extracorporeal endotoxin removal for the treatment of sepsis: endotoxin adsorption cartridge (Toraymyxin). The Apher Dial 7: 108-114

*Corresponding author: Toshihisa Ishikawa, RIKEN Yokohama Institute, RIKEN Omics Science Center, 1-7-22 Suehiro-cho, Tsurumi-ku Yokohama 230-0045, Japan, Tel: +81-503-9222; Fax: +81-503-9216; E-mail: toshi-i@gsc.riken.jp

Received November 26, 2012; Accepted November 27, 2012; Published Decembet 01, 2012

Citation: Ishikawa T (2012) Clinical Preparedness for Cytokine Storm Induced By the Highly Pathogenic H5N1 Influenza Virus. J Pharmacogenom Pharmacoproteomics 3: e131. doi:10.4172/2153-0645.1000e131

Copyright: (c) 2012 Ishikawa T. This is an open-access article distributed unde the terms of the Creative Commons Attribution License, which permits unrestricted use, distribution, and reproduction in any medium, provided the original author and source are credited. 